\title{
Sonographic assessment of the conventional 'blind' ilioinguinal block
}

\author{
Kiran Randhawa, MRCS · Soni Soumian, FRCS • \\ Maung Kyi, MBBS · Harmeet Khaira, FRCS
}

Received: 13 September 2009/Accepted: 14 October 2009/Published online: 24 October 2009

(C) Canadian Anesthesiologists' Society 2009

\section{To the Editor,}

Ilioinguinal nerve (IIN) and iliohypogastric nerve (IHN) block reduces the requirement for general anesthesia and opioid analgesics ${ }^{1}$ after inguinal hernia repair. Despite the conventional "blind" fascial click technique being practiced for a long period of time, a failure rate of $10-25 \%$ has been documented. ${ }^{2}$ The reasons suggested include anatomical variations, patient habitus (bulky subcutaneous tissues and well-developed muscles), and the inaccurate delivery of local anesthesia. Complications, such as femoral nerve blocks ${ }^{3}$ and infrequent injury to bowel and blood vessels, have also been reported. Ultrasound guidance to direct these blocks has been successful and is increasingly being adopted. ${ }^{4}$ In order to ascertain the reasons for the failure rate, we used ultrasound to assess the precision of needle placement in the "blind" technique.

We assessed preoperative IIN blocks performed by an anesthesiologist who had completed more than 200 nerve blocks on 21 subjects (19 males and two females; mean age 72 [range 43-93] yrs) undergoing inguinal hernia repair under general anesthesia. Institutional Research and Audit Department approval and informed consent from patients were obtained. The "blind" technique was performed as follows: Under aseptic precautions, a 22 gauge 1.50-in. regional block needle was used to pierce the skin two finger breadths medial to the anterior superior iliac spine. The correct layer was attained when the operator felt a "fascial click" on piercing the external oblique aponeurosis. A local

K. Randhawa and S. Soumian are contributed equally to the work.

K. Randhawa, MRCS · S. Soumian, FRCS $(\bowtie)$.

M. Kyi, MBBS - H. Khaira, FRCS

Good Hope Hospital, Birmingham, UK

e-mail: sonis@doctors.org.uk anesthetic was injected into this plane. Then the needle was passed medio-caudally and injections were made deeper between the internal oblique muscle (IOM) and the transversus abdominis muscle (TAM). This landmark was chosen because the IIN and the IHN would have penetrated the TAM at this location with a probability of 90 and $95 \%$, respectively. Both nerves may be found between the TAM and the IOM with $90 \%$ probability. ${ }^{5}$ Next, an observer experienced in ultrasound assessed the needle tip position (Sonosite $^{\mathrm{TM}}$ [MicroMAXX ${ }^{\circledR} /$ resolution $10-15 \mathrm{MHz}$ ] SonoSite, Inc., Bothell, WA, USA). The data recorded included the ability to visualize all muscle layers, peritoneum, nerves, and the needle tip (Fig. 1). If the needle tip was not visible, a small volume of levobupivacaine $5 \mathrm{mg} / \mathrm{mL}$ was injected to make evident the separation of tissue planes on the ultrasound monitor.

The muscle layers and the peritoneum were visualized in all subjects; however, the nerves were not visualized in any case. In 12 of the 21 subjects (57\%), the tip of the needle was positioned in the correct plane between the IOM and the TAM. In the other nine subjects, the needle tip was found deep to the TAM, and the potential failure rate was $43 \%$. None of the patients suffered complications, and all were discharged within $24 \mathrm{hr}$ after surgery.

Even with experience, the "blind" technique is likely to have a significant failure rate. In our study of the "blind" technique, the needle tip was deep to the TAM in $>40 \%$ of cases, which can potentially reduce the effectiveness of the block technique and increase the risk of complications. Our study did not assess the effectiveness of these blocks. The spread of the anesthetic has been considered the potential cause of complications such as femoral nerve blocks. A study in children reported an incidence of ipsilateral leg weakness postoperatively as high as $8.8 \% .^{3}$ Systematic understanding of anatomical structures, familiarity in 

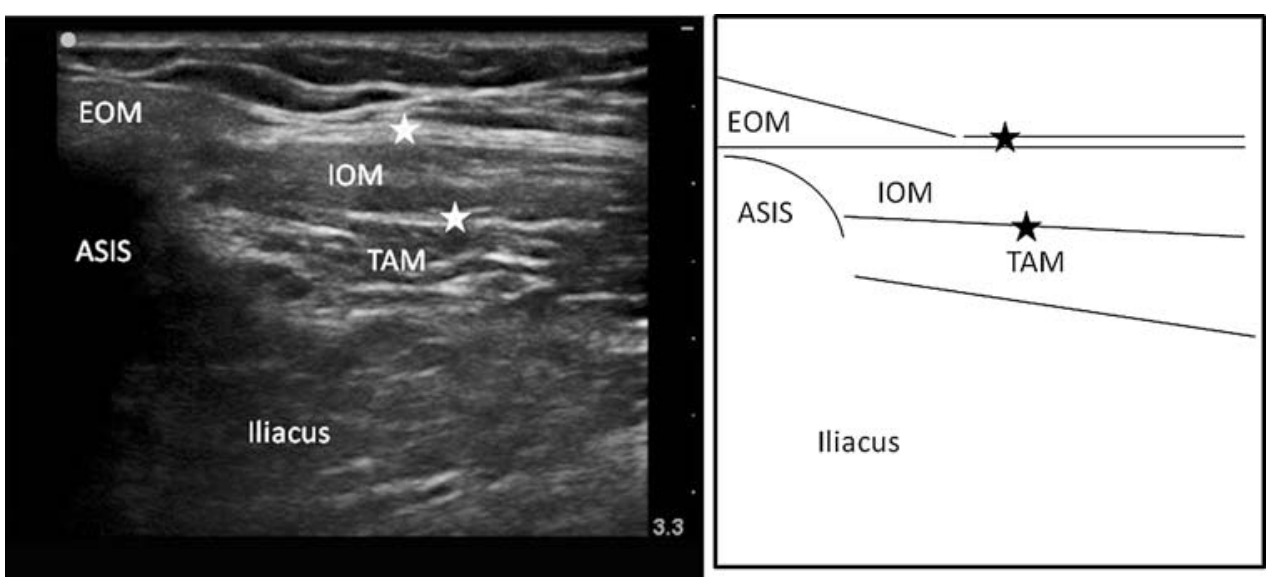

Fig. 1 Ultrasound image of the right abdominal wall in a transverse plane and corresponding pictogram. The two white stars mark the ideal injection sites where the iliohypogastric (between EOM and IOM) and ilioinguinal (between IOM and TAM) nerves traverse. The

ultrasound technology, and practical skills should be components of the armamentarium of anesthesiologists involved in regional anesthesia. Accurate visualization of the anatomy can increase success rates. Considering its potential, ultrasound can be a useful adjunct in IIN blocks.

\section{Conflicts of interest None declared.}

\section{References}

1. Bugedo GJ, Carcamo CR, Mertens RA, Dagnino JA, Munoz HR. Preoperative percutaneous ilioinguinal and iliohypogastric nerve depth of the image is $3.3 \mathrm{~cm}$. EOM external oblique muscle; $I O M$ internal oblique muscle; TAM transversus abdominus muscle; $A S I S$ anterior superior iliac spine

block with $0.5 \%$ bupivacaine for post-herniorrhaphy pain management in adults. Reg Anesth 1990; 15: 130-3.

2. van Schoor AN, Boon JM, Bosenberg AT, Abraham PH, Meiring $J H$. Anatomical considerations of the pediatric ilioinguinal/ iliohypogastric nerve block. Paediatr Anaesth 2005; 15: 371-7.

3. Lipp AK, Woodcock J, Hensman B, Wilkinson K. Leg weakness is a complication of ilio-inguinal nerve block in children. Br J Anaesth 2004; 92: 273-4.

4. Gofeld M, Christakis $M$. Sonographically guided ilioinguinal nerve block. J Ultrasound Med 2006; 25: 1571-5.

5. Jamieson RW, Swigart LL, Anson BJ. Points of parietal perforation of the ilioinguinal and iliohypogastric nerves in relation to optimal sites for local anaesthesia. Q Bull Northwest Univ Med Sch 1952; 26: $22-6$. 